\title{
What lies beneath: cutaneous Kaposi's sarcoma as a first manifestation of ectopic ACTH-dependent Cushing's syndrome
}

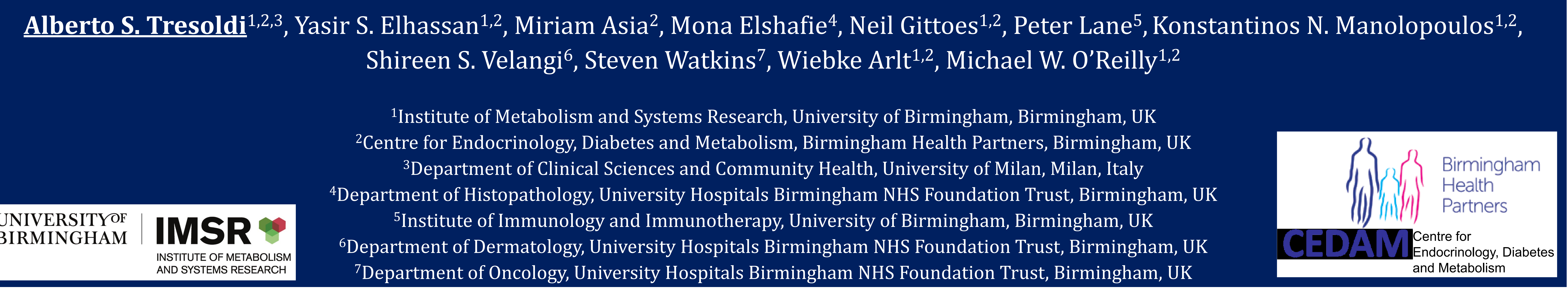

\section{Introduction}

Immune dysregulation is a feature of Cushing's syndrome (CS) [1]. Kaposi's sarcoma is an angioproliferative disorder that requires infection with human herpes virus 8 (HHV-8), and it is frequently an immunosuppression-related neoplasia [2].

We report a case of CS that presented with rapidly developing cutaneous Kaposi's sarcoma (KS).

\section{Case description}

A previously fit 59-year-old heterosexual man presented with a two-month history of proximal muscle weakness, recurrent mouth ulcers, and purplish skin lesions (Figure 1). He had a background history of hypertension. Skin biopsies were compatible with KS (Figure 2).

History of past residence in human herpesvirus 8 (HHV8) endemic countries was confirmed. Blood tests revealed T cell lymphopenia with low CD4+ and CD8+ lymphocytes and normal natural killer and $\mathrm{B}$ cells.

He underwent investigations in four hospital departments (neurology, dermatology, oncology and immunology) but no underlying predisposing factor was identified.

Four months later, centripetal obesity, facial plethora and dorsocervical fat pad were noted, which triggered a referral to the endocrine department. The clinical condition of the patient had deteriorated significantly, with an increase in the number of Kaposi's lesions which caused the patient to walk with a stick. Endocrine workup revealed an ACTH dependent hypercortisolism associated with secondary hypogonadism and elevated adrenal androgens (Table 1).

A pituitary MRI scan showed a lesion compatible with a microadenoma (Figure 3). However, both CRH test (Table 2) and inferior petrosal sinus sampling (Table 3) were consistent with an ectopic ACTH source.

Cross-sectional imaging, FDG-PET and ${ }^{68}$ Ga-DOTATE PET did not reveal any radiological evidence of a putative ACTH-producing lesion (Figure 4). Metyrapone treatment was commenced, and after a month bilateral adrenalectomy was performed.

Two weeks postoperatively the patient was able to stand up without a stick and to walk 150 yards. CD4 and CD8 counts normalized and hypertension resolved. Most of Kaposi's lesions completely resolved, and the biggest ones showed a reduction in maximum diameter (Figure 5).

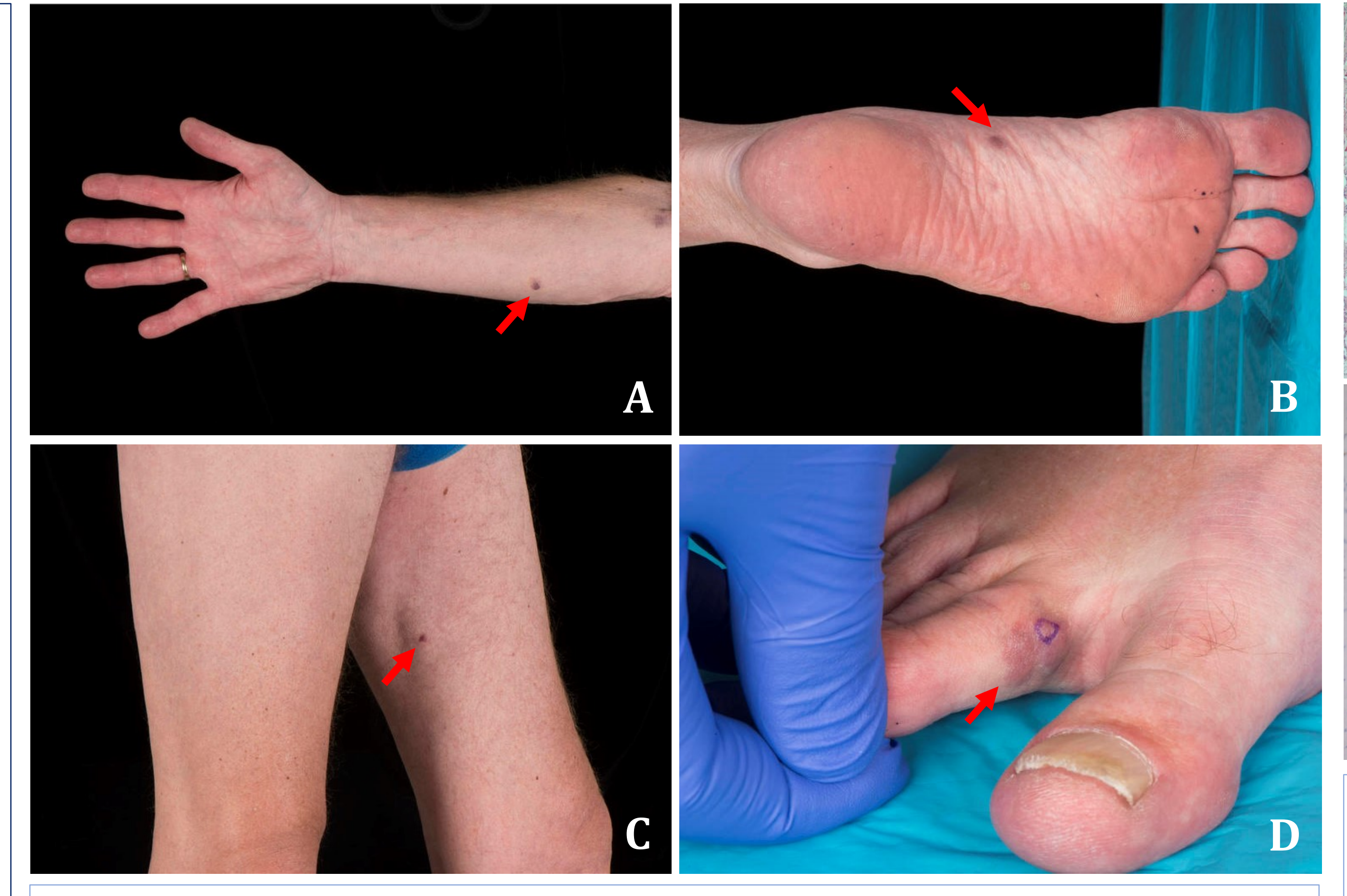

Figure 1. Purplish nodular cutaneous lesions with different locations (arrows). Right medial forearm (A), left sole of the foot (B), left medial thigh (C) and right foot second toe (D).

\begin{tabular}{|c|c|c|}
\hline Test & Result & Range \\
\hline 1mgDST & $794 \mathrm{nmol} / \mathrm{l}$ & $<50$ \\
\hline UFC & $1132 \mathrm{nmol} / 24 \mathrm{~h}$ & $<130$ \\
\hline ACTH & $100.3 \mathrm{ng} / \mathrm{l}$ & $7.2-63.3$ \\
\hline Androstenedione & $16.5 \mathrm{nmol} / \mathrm{l}$ & $0.8-4.7$ \\
\hline DHEA-S & $8.94 \mu \mathrm{mol} / \mathrm{l}$ & $1.4-8.01$ \\
\hline Testosterone & $3.6 \mathrm{nmol} / \mathrm{l}$ & $7.0-27.0$ \\
\hline FSH & $2.7 \mathrm{U} / \mathrm{l}$ & $1.5-12.4$ \\
\hline $\mathrm{LH}$ & $1.5 \mathrm{U} / \mathrm{l}$ & $1.7-8.6$ \\
\hline $\mathrm{K}+$ & $2.7 \mathrm{mEq} / 1$ & $3.5-5$ \\
\hline
\end{tabular}

Table 1. Endocrine work up

\begin{tabular}{|lllllll|}
\hline & $0^{\prime}$ & $1^{\prime}$ & $30^{\prime}$ & $4^{\prime}$ & $60^{\prime}$ & $90^{\prime}$ \\
\hline ACTH & 139.5 & 142.0 & 139.6 & 136.3 & 126.7 & 126.4 \\
\hline Cortisol & 1011 & 944 & 945 & 940 & 905 & 882 \\
\hline
\end{tabular}
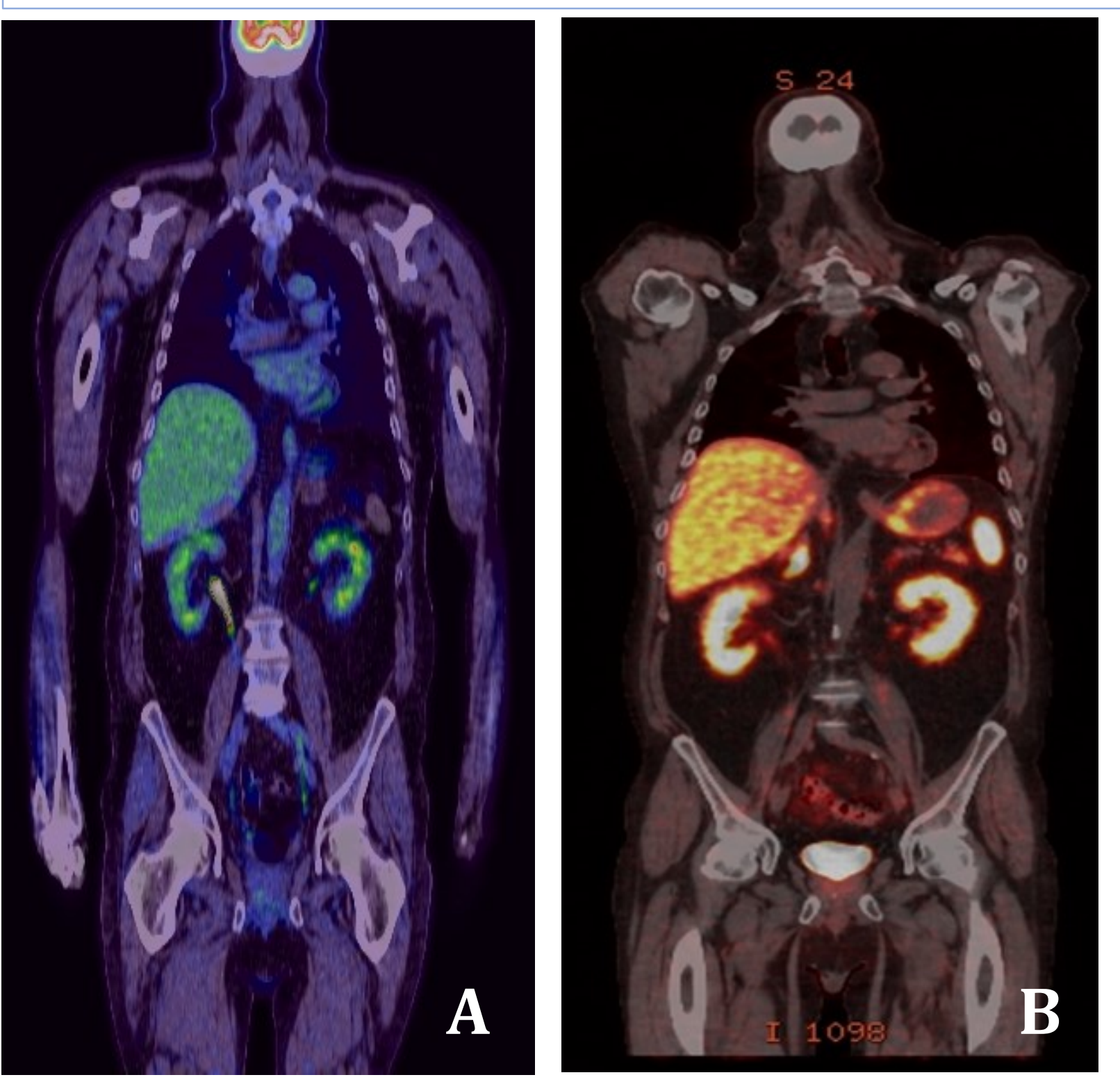

Figure 4. FDG-PET (A) and Ga68 DOTATATE-PET (B). No metabolic evidence of avid disease
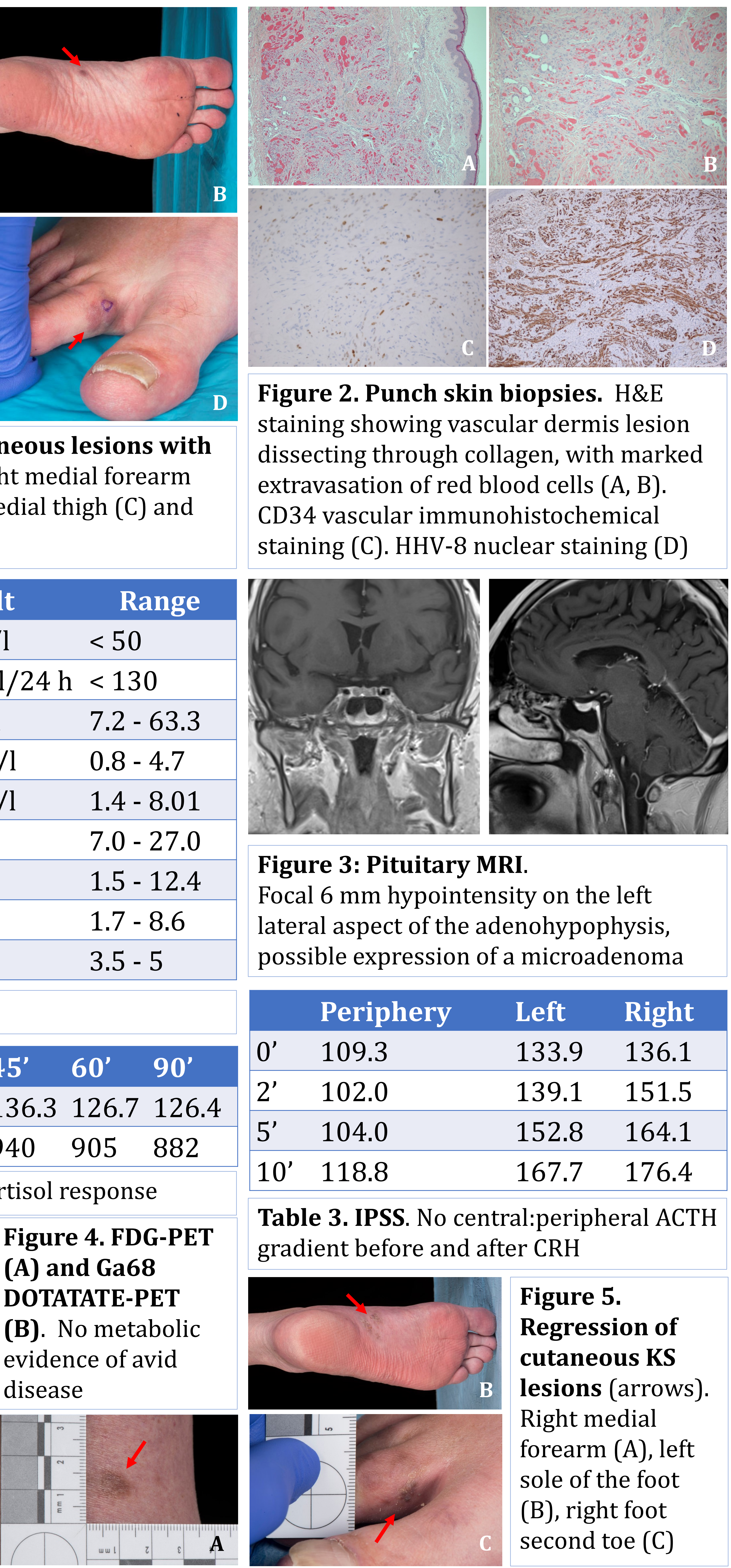

Figure 2. Punch skin biopsies. $H \& E$ staining showing vascular dermis lesion dissecting through collagen, with marked extravasation of red blood cells (A, B). CD34 vascular immunohistochemical staining (C). HHV-8 nuclear staining (D)

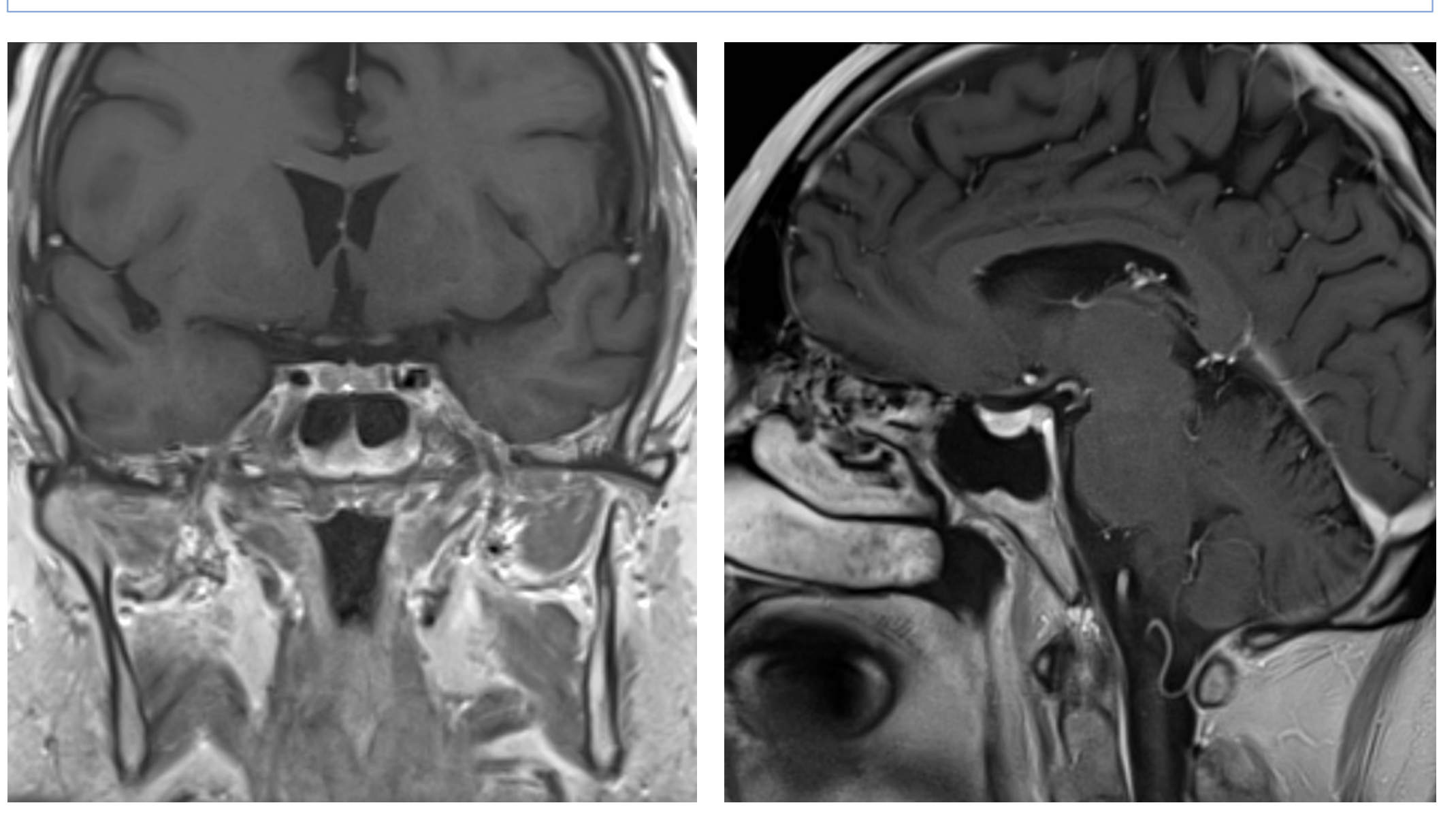

Figure 3: Pituitary MRI.

Focal $6 \mathrm{~mm}$ hypointensity on the left lateral aspect of the adenohypophysis, possible expression of a microadenoma

\begin{tabular}{|llll|} 
& Periphery & Left & Right \\
\hline $0^{\prime}$ & 109.3 & 133.9 & 136.1 \\
\hline $2^{\prime}$ & 102.0 & 139.1 & 151.5 \\
\hline $5^{\prime}$ & 104.0 & 152.8 & 164.1 \\
\hline $10^{\prime}$ & 118.8 & 167.7 & 176.4 \\
\hline
\end{tabular}

Table 3. IPSS. No central:peripheral ACTH gradient before and after $\mathrm{CRH}$

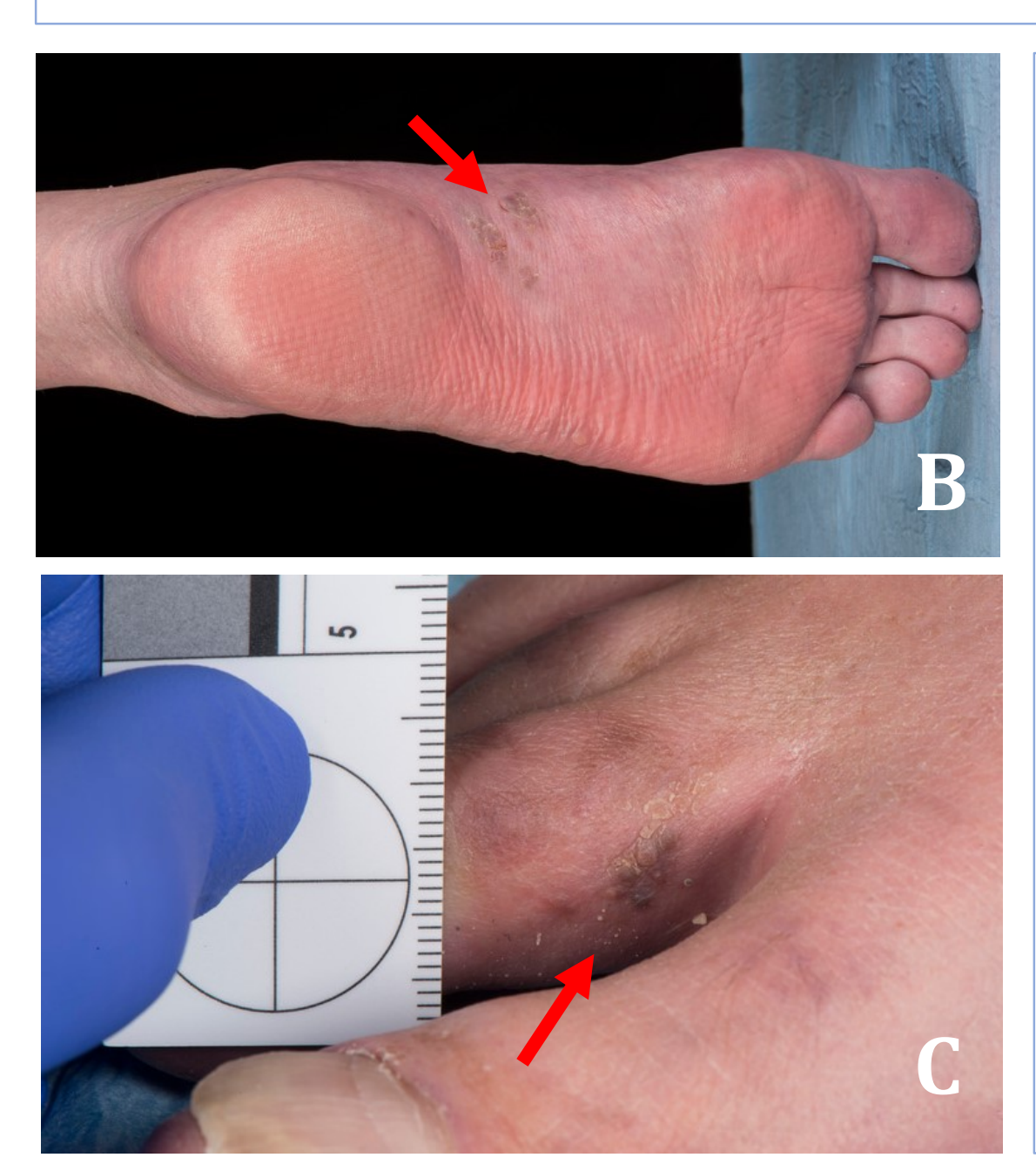

Figure 5. Regression of cutaneous KS lesions (arrows) Right medial forearm (A), left sole of the foot (B), right foot second toe $(\mathrm{C})$

\section{Conclusion}

Kaposi's sarcoma is an angioproliferative disorder related to HHV-8 infection. Severe immunodeficiency, such as AIDS or the use of immunosuppressant drugs, predisposes to KS.

We propose that Cushing's syndrome should be included in the differential diagnosis of immunodeficiencies causing KS. 